Taxonomía y sistemática

\title{
Crinoides (Echinodermata: Crinoidea) del Pensilvánico de sierra Las Mesteñas, NE de Sonora, México
}

\author{
Crinoids (Echinodermata: Crinoidea) of the Pennsylvanian from Sierra Las Mesteñas, NE \\ of Sonora, Mexico
Rafael Villanueva-Olea ${ }^{a}$, Blanca E. Buitrón-Sánchez ${ }^{b, *}$, Juan José Palafox-Reyes ${ }^{c}$ y Samael Piña-Flores ${ }^{\mathrm{d}}$

\footnotetext{
a Posgrado en Ciencias Biológicas, Universidad Nacional Autónoma de México, Av. Universidad 3000, Cd. Universitaria, 04510 Ciudad de México, México 04510 Ciudad de México, México

${ }^{\mathrm{c}}$ Departamento de Geología, Universidad de Sonora, Boulevard Luis Encinas y Rosales s/n, Col. Centro, 83000 Hermosillo, Sonora, México

${ }^{\mathrm{d}}$ Facultad de Ingeniería, Av. Universidad 3000, Cd. Universitaria, 04510 Ciudad de México, México

Recibido el 26 de febrero de 2016; aceptado el 26 de mayo de 2016

Disponible en Internet el 18 de noviembre de 2016
} \\ ${ }^{\mathrm{b}}$ Departamento de Paleontología, Instituto de Geología, Universidad Nacional Autónoma de México, Circuito de la Investigación Científica, Cd. Universitaria,
}

\begin{abstract}
Resumen
Se describen por primera vez 8 géneros y 9 especies de crinoides (Pentagonomischus plebeius, Axilinucrinus angustus, Cyclocaudex jucundus, Floricyclus angustimargo, Floricyclus granulosus, Cyclocrista cheneyi, Preptopremnum rugosum, Cycloscapus laevis y Cyclocaudiculus regularis), que proceden de afloramientos del Pensilvánico de sierra Las Mesteñas ubicados al noreste del estado de Sonora, México. Asociados a los crinoides, se identificaron briozoarios fenestélidos, braquiópodos de los géneros Anthracospirifer, Linoproductus, Composita, Cleiothyridina y Antiquatonia; corales solitarios (Lophophyllidium) y corales coloniales (Syringopora); esponjas coralinas (Chaetetes); foraminíferos fusulínidos (Fusulina, Triticites) y algas filoides (Komia, Eugonophyllum, Kamaena y Zidella). Esta biota se relacionó con otras del Pensilvánico de localidades de Sonora (Cerros El Tule y Sierra Agua Verde) y de Estados Unidos de América (Texas y Colorado). Asimismo, se infiere que la comunidad conformada por algas filoides incrustantes, fusulínidos, corales, briozoarios, braquiópodos y crinoides se desarrolló en un ambiente de plataforma carbonatada, de mares someros tropicales.
\end{abstract}

(C) 2016 Universidad Nacional Autónoma de México, Instituto de Biología. Este es un artículo Open Access bajo la licencia CC BY-NC-ND (http://creativecommons.org/licenses/by-nc-nd/4.0/).

Palabras clave: Caliza Naco; Encrinita; Plataforma carbonatada

\begin{abstract}
Eight genera and 9 species of crinoids are described for the first time (Pentagonomischus plebeius, Axilinucrinus angustus, Cyclocaudex jucundus, Floricyclus angustimargo, Floricyclus granulosus, Cyclocrista cheneyi, Preptopremnum rugosum, Cycloscapus laevis, and Cyclocaudiculus regularis); they proceed from outcrops of the Pennsylvanian of Sierra Las Mesteñas in northeastern Sonora, Mexico. Associated to the crinoids, fenestellid bryozoans; brachiopods of the genera Anthracospirifer, Linoproductus, Composita, Cleiothyridina, and Antiquatonia; solitary (Lophophyllidium) and colonial (Syringopora) corals; coralline sponges (Chaetetes); fusulinid foraminifera (Fusulina, Triticites); and phylloid algae (Komia, Eugonophyllum, Kamaena y Zidella) were identified. This biota is related with those from the Carboniferous of Sonora (Cerros El Tule and Sierra Agua Verde) and from the United States of America (Texas and Colorado). The community made up by encrusting phylloid algae, fusulinids, corals, bryozoans, brachiopods and crinoids developed in a carbonate platform, shallow tropical-water environment. (C) 2016 Universidad Nacional Autónoma de México, Instituto de Biología. This is an open access article under the CC BY-NC-ND license (http://creativecommons.org/licenses/by-nc-nd/4.0/).
\end{abstract}

Keywords: Naco limestone; Encrinite; Carbonate platform

\footnotetext{
* Autor para correspondencia.

Correo electrónico: blancab@unam.mx (B.E. Buitrón-Sánchez).

La revisión por pares es responsabilidad de la Universidad Nacional Autónoma de México.
}

http://dx.doi.org/10.1016/j.rmb.2016.10.014

1870-3453/@ 2016 Universidad Nacional Autónoma de México, Instituto de Biología. Este es un artículo Open Access bajo la licencia CC BY-NC-ND (http://creativecommons.org/licenses/by-nc-nd/4.0/). 


\section{Introducción}

Las investigaciones sobre el Paleozoico de México son escasas, particularmente sobre la sierra Las Mesteñas. Entre los estudios realizados en esta localidad, destaca el desarrollado por Gómez-Tagle (1967), donde se indica de manera general la existencia de algunos fósiles de la región. Stewart, McMenamin y Morales-Ramírez (1984) documentaron la geología de varias localidades paleozoicas de Sonora. Peiffer-Rangin (1987) estudió diversas secuencias paleozoicas en el noreste y en el centro de Sonora, entre ellas la sierra Las Mesteñas, haciendo una descripción detallada de la estratigrafía, así como descripciones paleontológicas. Page et al. (2010) realizaron el estudio sobre la geología en sierra Los Ajos, adyacente a Las Mesteñas, revelando nuevos datos estructurales y estratigráficos del área. En la secuencia de estratos del Pensilvánico que aflora en la localidad de estudio, se había identificado previamente una gran variedad de invertebrados fósiles correspondientes a briozoarios fenestélidos, braquiópodos espiriféridos (Punctospirifer) y prodúctidos (Antiquatonia), corales coloniales (Michelinia) como solitarios (Lophophyllidium), fusulínidos (Fusulina) y numerosos fragmentos de crinoides no descritos anteriormente.

Desde el punto de vista geológico, el basamento en el área está constituido por la unidad Esquisto Pinal, fechada en 1,640 Ma, que aflora en la porción sur occidental y está intrusionado por el Granito Mesteñas.

El Paleozoico para la región noreste de Sonora presenta facies de plataforma y varía en edad del Cámbrico medio al Pérmico temprano, con hiatus entre el Cámbrico superior al Devónico temprano (Gómez-Tagle, 1967; Taliaferro, 1933). En el área de Las Mesteñas se inicia la depositación de la Cuarcita Bolsa y Formación Abrigo del Cámbrico medio al superior. La Cuarcita Bolsa, definida por Ransome (1904), se caracteriza por presentar horizontes de arenisca con clastos de cuarzo de pocos milímetros, además de estratificación cruzada y una capa de conglomerados en su base. Los estratos de la Cuarcita Bolsa subyacen a los de la Caliza Abrigo del Cámbrico superior mediante un contacto transicional (Ransome, 1904). Esta formación se constituye de caliza gris oscuro de estratos delgados a medios y caliza margosa amarilla, de estratificación laminar con crinoides y braquiópodos (Gómez-Tagle, 1967).

En discordancia erosional, se encuentra la secuencia representada por la Caliza Martin del Devónico (Ransome, 1904), con 2 miembros: el inferior, constituido por caliza gris oscura, de estratificación media, con crinoides, y el superior, compuesto por caliza gris-oscura, de estratificación delgada, con lentes de pedernal y sin fósiles (Gómez-Tagle, 1967). Sobreyaciendo, en contacto concordante, se ubica la Caliza Escabrosa del Misisípico, constituida por caliza de color gris-claro, de aspecto granuloso y de estratificación gruesa, con crinoides y braquiópodos como fauna principal (Gómez-Tagle, 1967).

Por encima se localizan, de manera concordante, los estratos de la Caliza Naco (Ransome, 1904) del Pensilvánico. Estos corresponden a caliza gris claro, de estratificación delgada a media, con presencia, en la parte superior de caliza arenosa con abundantes placas columnares de crinoides. Por encima de la Caliza Naco, en discordancia angular, se ubican rocas del Cretácico (Gómez-Tagle, 1967).

En el presente estudio se hizo la determinación paleontológica del material de columnas de crinoides y placas columnares procedentes de rocas del Pensilvánico de la sierra Las Mesteñas, que consta de 8 morfogéneros y 9 morfoespecies, de acuerdo con el sistema de clasificación parataxonómica propuesto por Moore y Jeffords (1968).

\section{Materiales y métodos}

La sierra Las Mesteñas $\left(30^{\circ} 58^{\prime}-31^{\circ} 05^{\prime} \mathrm{N}, 109^{\circ} 45^{\prime}-108^{\circ} 52^{\prime}\right.$ O) se encuentra localizada en el noreste del estado de Sonora, en la región de Cananea, aproximadamente a $45 \mathrm{~km}$ al suroeste de la ciudad de Agua Prieta (fig. 1). La sierra se ubica dentro de la provincia fisiográfica de la Sierra Madre Occidental, dentro de la subprovincia Zona de Sierras y Valles Paralelos; alrededor de Las Mesteñas, se encuentran otras unidades orográficas de gran importancia, como las sierras Los Ajos, Cánovas y Las Cenizas, entre otras (Gómez-Tagle, 1967). En la región noreste de la sierra Las Mesteñas se encuentra expuesta una secuencia estratigráfica de rocas de plataforma marina del Pensilvánico con un espesor total de $300 \mathrm{~m}$ (fig. 2). A continuación, se describe la litología en intervalos de $30 \mathrm{~m}$ desde la parte inferior hasta la superior: 1-30 m: caliza en estratos gruesos de colores que varían desde gris a café oscuro. Se identificó la siguiente fauna fósil: placas columnares de crinoides pequeños, circulares y pentagonales, briozoarios fenestélidos, braquiópodos (Antracospirifer, Cleiothyridina), corales (Lophophyllidium) y fusulínidos; 30-60 m: caliza de color café claro con estratificación delgada, incluye también caliza de grano fino con escasos zaphréntidos, numerosos crinoides, braquiópodos (Anthrascospirifer y Composita), escasos fusulínidos, gasterópodos, briozoarios ramosos y algas; 60-90 m: estratos delgados de color café claro a crema de caliza arcillosa con placas de crinoides circulares, pentagonales y pentalobados; briozoarios fenestélidos; corales coloniales (Syringopora) y solitarios zaphréntidos; esponjas del género Chaetetes; braquiópodos espiriféridos y prodúctidos (Linoproductus); fusulínidos y algas; 90-120 m: caliza en estratos delgados y gruesos, con intercalación de caliza arcillosa de color café claro a gris; se encontraron arrecifes de esponjas del género Chaetetes, braquiópodos espiriféridos y prodúctidos, crinoides y corales coloniales (Syringopora) y solitarios zaphréntidos, así como foraminíferos fusulínidos; 120-150 m: estratos de caliza delgados a medianos, intercalados por caliza arcillosa, con escasa fauna fósil, que consiste en numerosas placas de crinoides (encrinita), briozoarios fenestélidos y braquiópodos espiriféridos; 150-180 m: estratos de caliza delgados a medianos de colores café claro a gris, intercalados por caliza arcillosa de color café claro, fauna fósil escasa, encontrándose corales, crinoides (encrinita), fragmentos de braquiópodos prodúctidos como Antiquatonia, restos de briozoarios fenestélidos y tubos de gusanos; 180-210 m: caliza en estratos medianos a gruesos que varía de color café claro a gris, presentando en ocasiones intercalaciones de caliza arcillosa. En este intervalo se observó biota fósil muy escasa de algas, crinoides, briozoarios ramosos, escasos fragmentos de braquiópodos espiriféridos y restos de 


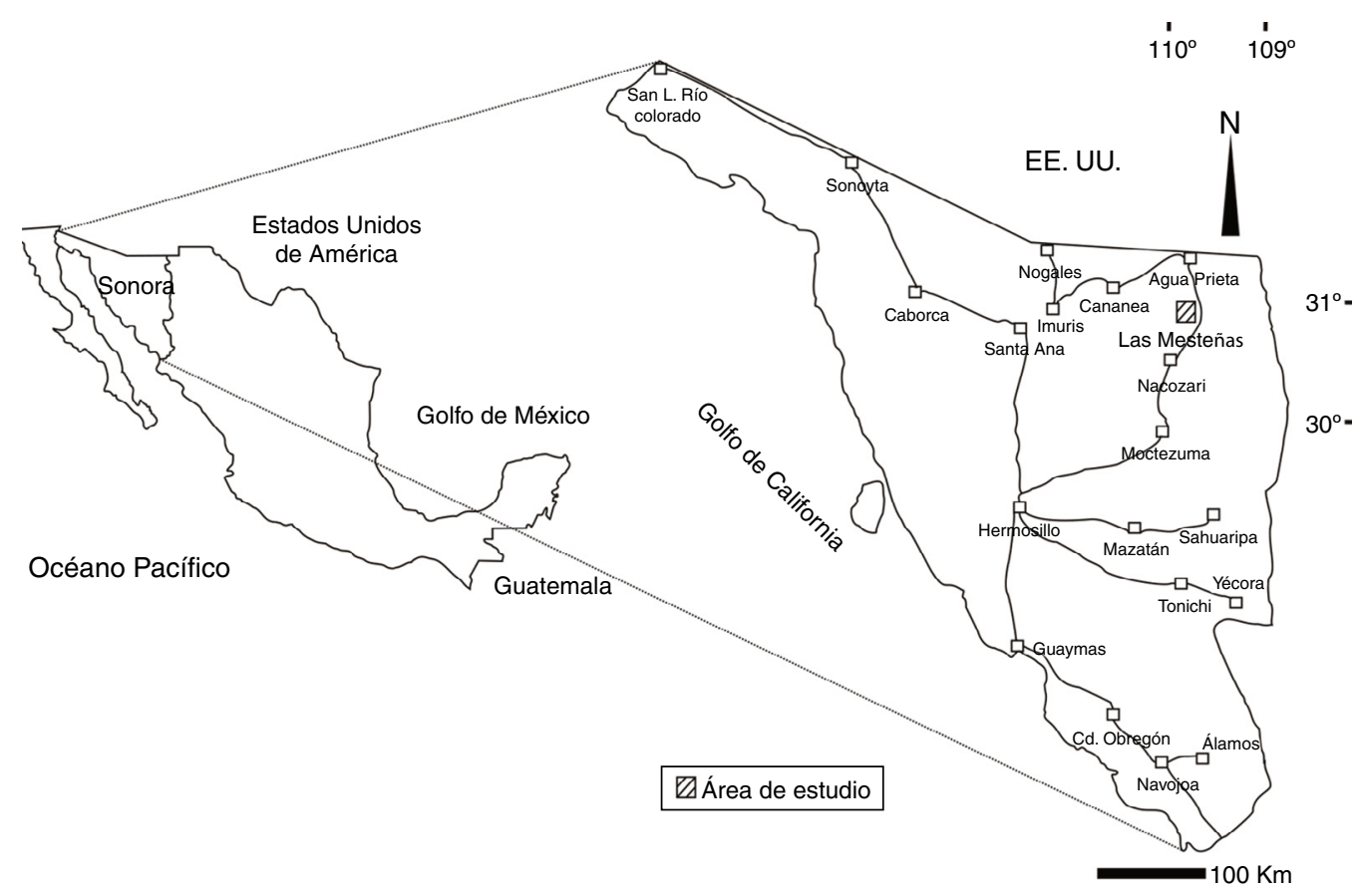

Figura 1. Localización de sierra Las Mesteñas en el estado de Sonora, México.

corales de gran tamaño, así como corales solitarios zaphréntidos; 210-240 m: caliza con estratificación mediana a gruesa de color café a gris claro; además de encrinitas, se recolectaron braquiópodos bien conservados, corales solitarios, briozoarios fenestélidos y esponjas (Chaetetes); 240-270 m: caliza de estratificación delgada a mediana de color gris claro y café claro con encrinita. Se observaron braquiópodos prodúctidos (Antiquatonia) y espiriféridos; $270-300 \mathrm{~m}$ : caliza de estratificación delgada a mediana de color gris claro y café claro. Se observaron encrinitas, briozoarios ramosos, restos de braquiópodos espiriféridos y prodúctidos.

La edad para esta secuencia, con base en los crinoides identificados, se definió en Pensilvánico (Carbonífero), correspondiente a la Caliza Naco del Pensilvánico, que Ransome (1904) identificó por primera vez en el Cuadrángulo de Bisbee en Arizona, y en Sonora fue identificada por Taliaferro (1933). Un estudio con base en foraminíferos podría afinar la edad a nivel de piso para estos estratos.

El material fósil de crinoides recolectado consistió principalmente en numerosas placas columnares, que presentan una morfología característica que permitió su determinación a nivel específico. El material se fotografió a aumentos variables de acuerdo con su tamaño; para ello, se siguió la técnica de recubrimiento con cloruro de amonio con el fin de contrastar el ejemplar y destacar las diferentes estructuras. Las fotografías fueron tomadas con una cámara analógica Nikon F3 con una lente Micro de $50 \mathrm{~mm}$. El material fue identificado y clasificado de acuerdo con Moore y Jeffords (1968) y con Ubaghs et al. (1978). El material fósil estudiado está depositado en la Colección Nacional de Paleontología del Instituto de Geología de la UNAM. Los ejemplares tienen número de catálogo provisional IGM-4868 - IGM-4876.

\section{Descripción}

Clase Crinoidea Miller, 1821

Subclase y Orden inciertos Moore y Jeffords, 1968

[Grupo Pentameri] Moore y Jeffords, 1968

Familia Pentacauliscidae Moore y Jeffords, 1968

Género Pentagonomischus Moore y Jeffords, 1968

Diagnosis: columna pentagonal, heteromórfica; crénulas cortas, gruesas y rectas; aréola amplia y lisa a ligeramente cóncava que se extiende hacia un lumen circular, grande y sin borde; el canal axial contiene un claustro delgado a la mitad de cada placa columnar, circundado por un yúgulo pequeño y estelado.

Especie tipo: Pentagonomischus plebeius Moore y Jeffords, 1968, págs. 53-54, lám. 8.

Pentagonomischus plebeius Moore y Jeffords, 1968(fig. 3)

Pentagonomischus plebeius Moore y Jeffords, 1968, págs. 53-54, lám. 8

Pentagonomischus cf. P. plebeius Villanueva-Olea, CastilloEspinoza, Sour-Tovar, Quiroz-Barroso y Buitrón-Sánchez, 2011, p. 447, figura 3 a-c

Descripción. La placa es de contorno pentagonal; el crenulario presenta crénulas muy anchas y rectas, de tamaño variable, que dan forma a la aréola, la cual es petaloide y con numerosos gránulos; no se observa un patrón de $\mathrm{Y}$ o $\mathrm{V}$ en la forma de las crénulas, que es típica en placas de aréola petaloide o estelar; el lumen es grande y de apariencia circular.

Distribución. P. plebeius se reporta para el Chesteriano (Misisípico Superior) de la Formación Paint Creek de Illinois, EE. UU. (Moore y Jeffords, 1968). En México, se reporta el género Pentagonomischus para el Misisípico Inferior de San Salvador Patlanoaya, Puebla (Villaseñor-Martínez, MartínezCortés y Contreras y Montero, 1987) y P. cf. P. plebeius 

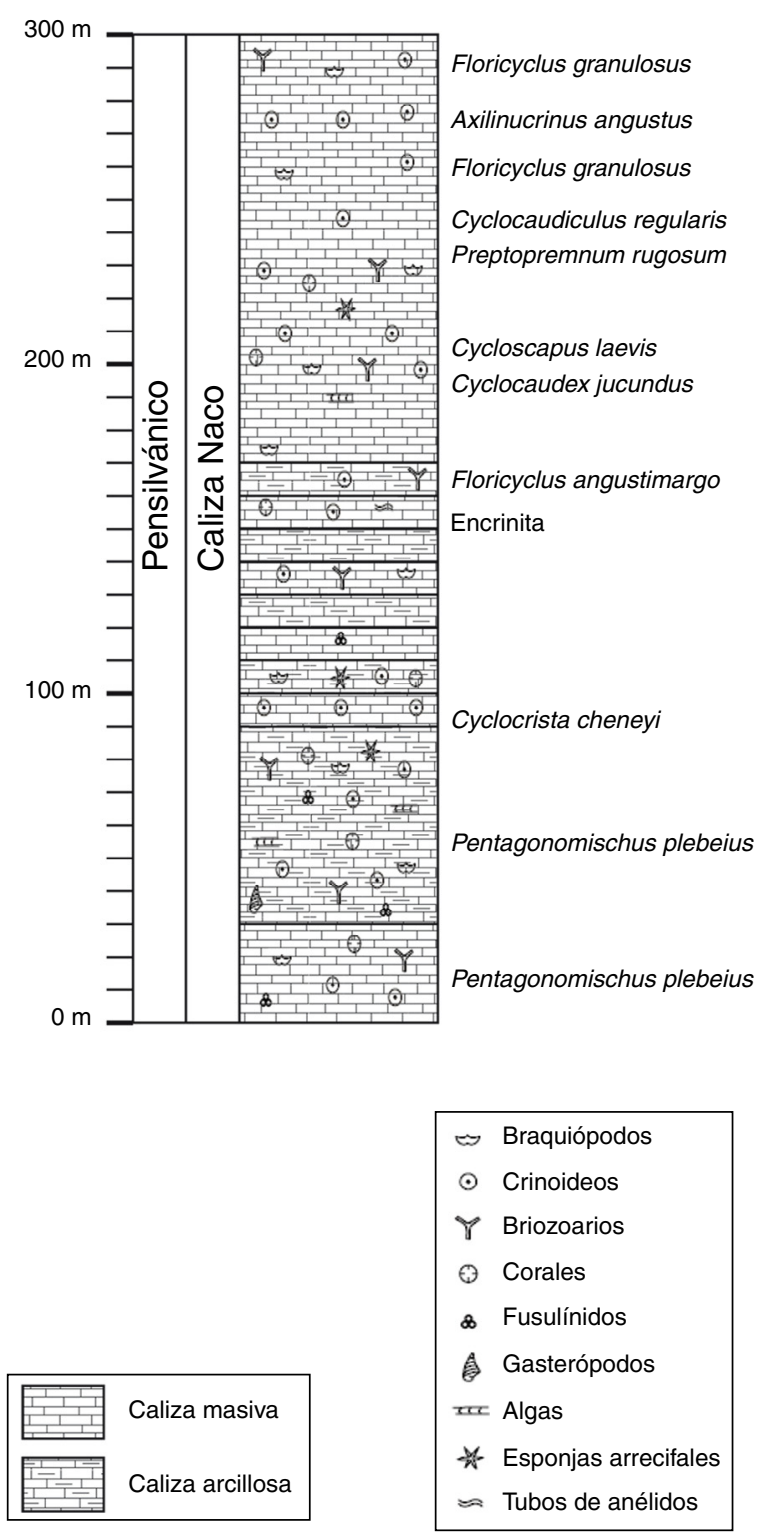

Figura 2. Columna bioestratigráfica del Pensilvánico de sierra Las Mesteñas, Sonora, México.

para el Morrowano-Desmoinesiano (Pensilvánico) de Santiago Ixtaltepec, Oaxaca (Villanueva-Olea et al., 2011).

Número de catálogo: IGM-4868.

Comentarios taxonómicos

Esta especie fue comparada con el género Pentaridica del Pensilvánico Medio-Superior de EE. UU. (Moore y Jeffords, 1968), del cual se distingue por tener la aréola estelar y estrecha, mientras que en las diferentes especies de Pentaridica, la aréola es de contorno pentagonal y se hunde hacia el interior del lumen.

\section{[Grupo Cyclici]}

Familia Cyclomischidae Moore y Jeffords, 1968

Género Axilinucrinus Villanueva-Olea et al., 2011

Diagnosis: las placas articulares del género presentan crénulas rectas y crenulario circular y estrecho.
Especie tipo: Axilinucrinus angustus Villanueva-Olea et al., 2011, págs. 449-450, figura 3.

Axilinucrinus angustus Villanueva-Olea et al., 2011

(fig. 4)

Axilinucrinus angustus Villanueva-Olea et al., 2011, págs. 449-450, figura 3

Descripción. La placa es de contorno circular; el crenulario moderadamente ancho con crénulas rectas, gruesas, sin bifurcaciones; no presenta aréola, por lo que el crenulario está en contacto directo con el lumen de forma ligeramente ovalada y de tamaño grande, ocupando un diámetro que es aproximadamente la mitad del diámetro de la faceta.

Distribución. A. angustus se reportó como género y especie nueva para el Morrowano-Desmoinesiano (Pensilvánico Inferior-Medio) de la Formación Ixtaltepec de Santiago Ixtaltepec, Oaxaca, México (Villanueva-Olea et al., 2011).

Localidad: sierra Las Mesteñas.

Número de catálogo: IGM-4869.

Comentarios taxonómicos

El ejemplar se comparó con otras especies de la familia Cyclomischidae como Rhysocamax, del Misisípico Inferior de Iowa, EE. UU. (Moore y Jeffords, 1968), del cual se diferencia por tener el lumen más grande y ovalado. Lomalegnum, del Misisípico Inferior de Iowa (Moore y Jeffords, 1968) también es parecido a la especie de sierra Las Mesteñas, excepto por el rasgo de que las crénulas en Lomalegnum son muy delgadas y muchas de ellas están bifurcadas.

\section{Género Cyclocaudex Moore y Jeffords, 1968}

Diagnosis: forma circular de la placa, crenulario ancho con crénulas largas y rectas, aréola pequeña, a veces faltante, o con el límite interno con características del perilumen; lumen típicamente circular y pequeño, pero puede ser subpentagonal a levemente quinquelobulado.

Especie tipo: Cyclocaudex typicus Moore y Jeffords, 1968, pág. 65, lám. 17.

Cyclocaudex jucundus Moore y Jeffords, 1968

(figs. 5 y 6 )

Cyclocaudex jucundus Moore y Jeffords, 1968; MorenoCano y Patiño-Ruiz, 1981, pág. 26, lám. 2, figura 3

Buitrón-Sánchez, Patiño y Moreno, 1987, pág. 132, figura 5; Velasco-de León y Buitrón-Sánchez, 1992, pág. 75, lám. 2, figuras 1-4; Buitrón-Sánchez y Solís-Marín, 1993, pág. 214; Arellano-Gil, Vachard, Yussim y Flores de Dios-González, 1998, pág. 11; Esquivel-Macías, Ausich, Buitrón-Sánchez y Flores de Dios, 2000, pág. 1188; Esquivel-Macías, SolísMarín y Buitrón-Sánchez, 2004, págs. 17, 19; Buitrón-Sánchez et al., 2008, págs. 3, 6, 7; Buitrón-Sánchez, Vachard, AlmazánVázquez y Palafox-Reyes, 2012, pág. 43, figura 7.18.

Cyclocaudex cf. C. (col.) jucundus Moore y Jeffords, 1968; Buitrón-Sánchez, Almazán-Vázquez y Vachard, 2007, pág. 347, figura $3 \mathrm{c}-\mathrm{d}$

Descripción. La placa columnar es de contorno circular con crenulario de tamaño moderado y crénulas muy gruesas y rectas; los cúlmenes son más anchos que las crenelas; la aréola es granulosa y de tamaño moderado, en contacto con el perilumen 

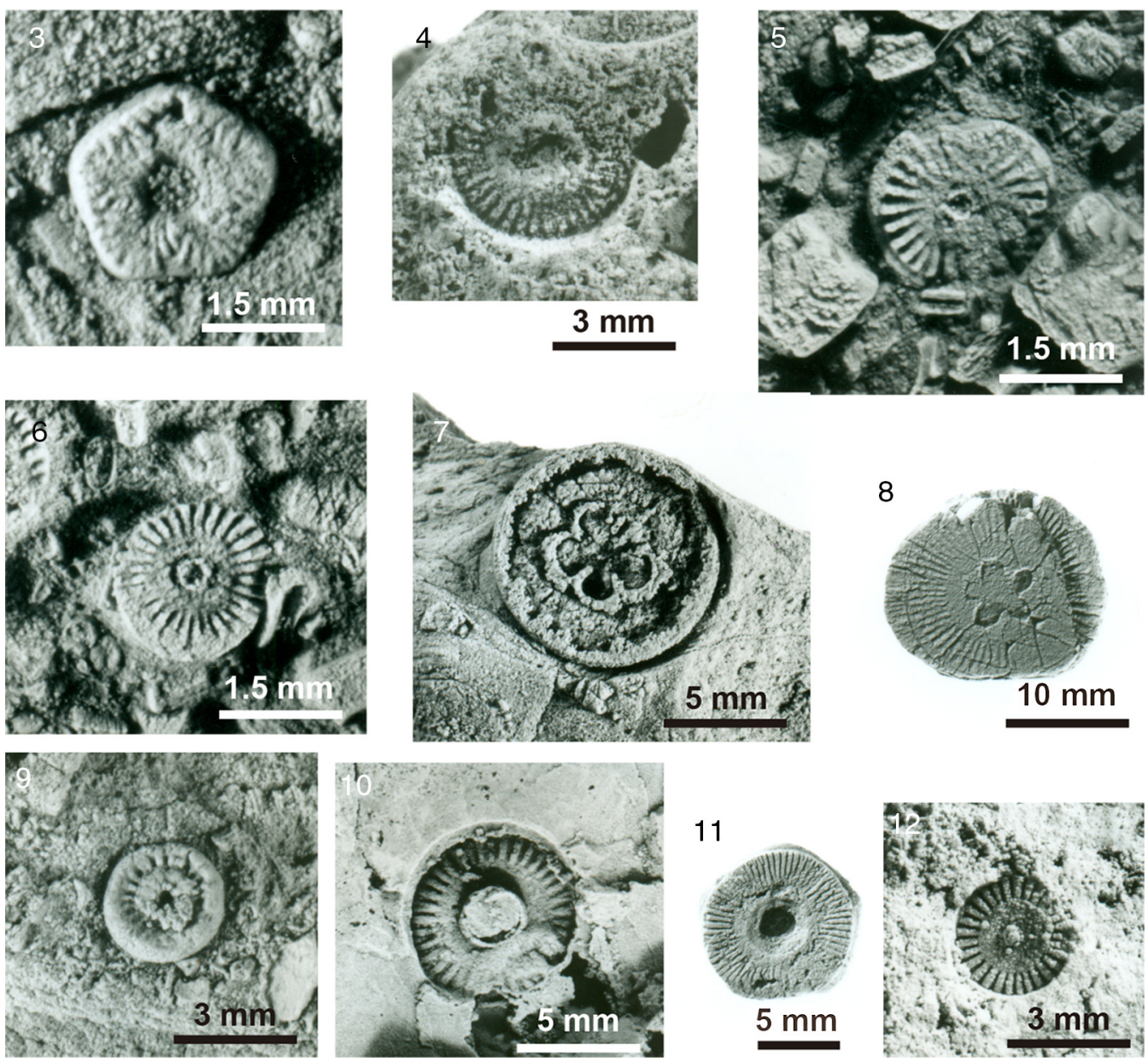

Figuras 3-12. Crinoides del Pensilvánico de sierra Las Mesteñas, Sonora.3: Pentagonomischus plebeius Moore y Jeffords, 1968. 4: Axilinucrinus angustus VillanuevaOlea et al., 2011. 5 y 6: Cyclocaudex jucundus Moore y Jeffords, 1968. 7: Floricyclus angustimargo Moore y Jeffords, 1968: 8: Floricyclus granulosus Moore y Jeffords, 1968. 9: Cyclocrista cheneyi Moore y Jeffords, 1968. 10: Preptopremnum rugosum Moore y Jeffords, 1968. 11: Cycloscapus laevis Moore y Jeffords, 1968. 12: Cyclocaudiculus regularis Moore y Jeffords, 1968.

muy estrecho que forma un borde alrededor del lumen, el cual es circular y pequeño.

Distribución. Cyclocaudex jucundus se reporta para la Formación Thrifty, del Virgiliano (Pensilvánico Superior) de Texas, EE. UU. (Moore y Jeffords, 1968). En México se ha citado para la Formación Del Monte, de edad pensilvánica, en Calnali, Hidalgo (Buitrón-Sánchez et al., 1987), para el Virgiliano (Pensilvánico Superior) de San Salvador Patlanoaya, Puebla (Velasco-de León y Buitrón-Sánchez, 1992), para el Pérmico inferior de la Formación Guacamaya de Pemuxco, Hidalgo (Arellano-Gil et al., 1998). C. jucundus también se reporta para el Pérmico medio de Cerros Los Monos, Sonora (BuitrónSánchez, Almazán-Vázquez et al., 2007); igualmente, para el Pensilvánico Medio-Superior (Desmoinesiano-Misuriano) de Cerros El Tule, Sonora (Buitrón-Sánchez et al., 2008, 2012).

\section{Números de catálogo: IGM-4870 e IGM-4871.}

\section{Comentarios taxonómicos}

El ejemplar de la sierra Las Mesteñas se asigna a $C$. jucundus debido a la forma del crenulario, a la presencia de un perilumen delgado, así como de una aréola que es más ancha que en otras especies del género. También se comparó con Cyclocrista cheneyi del Wolfcampiano (Pérmico inferior) de Texas (Moore y Jeffords, 1968), pero esta última tiene una aréola cóncava y el crenulario muy estrecho con crénulas más anchas que en el ejemplar de Sonora.

Familia Floricyclidae Moore y Jeffords, 1968

Género Floricyclus Moore y Jeffords, 1968

Diagnosis: faceta articular con lumen grande, pentalobulado a floriforme con gránulos pequeños entre los lóbulos petaloides, presencia de perilumen, aréola estrecha y el crenulario con crénulas cortas y moderadamente gruesas.

Especie tipo: Floricyclus hebes Moore y Jeffords, 1968, pág. 77, lám. 24.

Floricyclus angustimargo Moore y Jeffords, 1968

(fig. 7)

Floricyclus angustimargo Moore y Jeffords, 1968; EsquivelMacías et al., 2000, pág. 1188; Esquivel-Macías et al., 2004, pág. 17.

Descripción. La placa presenta contorno circular; el lumen es grande y floriforme, el perilumen es grueso y presenta gránulos alrededor de los lóbulos petaloides del lumen. El crenulario y la aréola están erosionados. 
Distribución. Floricyclus angustimargo se encuentra en la Formación Minturn, Desmoinesiano (Pensilvánico Medio) de Colorado, EE. UU.. En México, se registra para el Pensilvánico Medio-Superior de San Salvador Patlanoaya, Puebla (EsquivelMacías et al., 2000).

Localidad: sierra Las Mesteñas.

Número de catálogo: IGM-4872.

Comentarios taxonómicos

Se comparó el ejemplar con Floricyclus granulosus del Pensilvánico Superior de Texas (Moore y Jeffords, 1968), pero esta última especie presenta el lumen comparativamente más estrecho. Particularmente, las características del perilumen en el espécimen de sierra Las Mesteñas permitió su identificación con $F$. angustimargo.

Floricyclus granulosus Moore y Jeffords, 1968

(fig. 8)

Floricyclus granulosus Moore y Jeffords, 1968, pág. 77, láms. 24 y 26

Descripción. La placa está ligeramente deformada, pero presumiblemente de contorno circular; el crenulario es ancho y ocupa aproximadamente la mitad del diámetro de la faceta articular; las crénulas son muy delgadas y rectas; la aréola es muy estrecha y con pocas granulaciones; el lumen es relativamente pequeño, de apariencia floriforme, y no se observa un desarrollo evidente de perilumen.

Distribución. F. granulosus se distribuye en el Virgiliano (Pensilvánico Superior) de Texas, Formación Thrifty (Moore y Jeffords, 1968).

Número de catálogo: IGM-4873.

Comentarios taxonómicos

Algunas de las placas articulares de F. granulosus ilustradas en Moore y Jeffords (1968) muestran que la especie tiene escasas granulaciones, como en el ejemplar de sierra Las Mesteñas; también la ausencia del perilumen es evidente como en otras especies de Floricyclus. El lumen más pequeño y las crénulas más largas que en otras especies del género permitieron asignar este material a $F$. granulosus.

\section{Familia Leptocarphiidae Moore y Jeffords, 1968}

Género Cyclocrista Moore y Jeffords, 1968.

Diagnosis: faceta articular con crenulario delgado, crénulas cortas, gruesas y rectas, separadas de la aréola por un declive. La aréola es ligeramente cóncava con perilumen aparente muy ancho alrededor del lumen circular o quinquelobulado.

Especie tipo: Cyclocrista lineolata Moore y Jeffords, 1968, pág. 80, lám. 25.

Cyclocrista cheneyi Moore y Jeffords, 1968

(fig. 9)

Cyclocrista cheneyi Moore y Jeffords, 1968, pág. 80, lám. 26; Arellano-Gil et al., 1998, pág. 11; Esquivel-Macías et al., 2000, pág. 1188

Descripción. La placa articular es de forma circular con epifaceta muy gruesa de perfil convexo, que sobresale del crenulario, el cual se encuentra hundido en la placa. El crenulario es estrecho con crénulas muy gruesas y rectas. La aréola es cóncava y granulosa. Presenta el lumen circular muy pequeño.
Distribución. C. cheneyi se ha reportado para el Wolfcampiano (Pérmico inferior) de Texas (Moore y Jeffords, 1968). En México, se reporta para el Pérmico inferior (Leonardiano) de Pemuxco, Hidalgo (Arellano-Gil et al., 1998), y para el Pensilvánico Superior (Misuriano-Virgiliano) de la Formación Patlanoaya, San Salvador Patlanoaya, Puebla (Esquivel-Macías et al., 2000).

Número de catálogo: IGM-4874.

Comentarios taxonómicos

El material de Las Mesteñas se comparó con Preptopremnum laeve del Pensilvánico Medio de Texas (Moore y Jeffords, 1968) pero en el ejemplar estudiado, la epifaceta es conspicua. También se relacionó con Cyclocrista martini (Moore y Jeffords, 1968), sin embargo, el crenulario es mucho más ancho. De igual manera, en $C$. lineolata, del Pensilvánico Superior de Texas (Moore y Jeffords, 1968), no es evidente una epifaceta como en la especie estudiada.

Género Preptopremnum Moore y Jeffords, 1968

Diagnosis: crenulario estrecho con crénulas de tamaño medio a grueso. Aréola plana a ligeramente cóncava o convexa, marcada por irregularidades granulosas finas o vermiculadas. Lumen grande, circular, bordeada por un perilumen estrecho con claustro a media altura y yúgulo estelar diminuto.

Especie tipo: Preptopremnum rugosum Moore y Jeffords, 1968, pág. 81, láms 27-28.

Preptopremnum rugosum Moore y Jeffords, 1968

(fig. 10)

Preptopremmum rugosum Moore y Jeffords, 1968; ArellanoGil et al., 1998, pág. 11; Buitrón-Sánchez, Gómez-Espinosa, Almazán-Vázquez y Vachard, 2007, pág. 204, figura 3h; Buitrón-Sánchez, Almazán-Vázquez, 2007, pág. 347, figura 3 f, g.; Buitrón-Sánchez et al., 2008, págs. 6 y 7; González-Arreola, Villaseñor-Martínez y Corona-Esquivel, 1994, pág. 217, lám. 2, figura d; Buitrón-Sánchez et al., 2012, pág. 46, figura 7.21; Buitrón-Sánchez et al., 2015, pág. 523, figura 26.

Preptopremnum? cf. P. rugosum Moore y Jeffords, 1968; Esquivel-Macías et al., 2000, pág. 1188.

Descripción. La placa es de contorno circular con un crenulario mediano que presenta crénulas gruesas. La aréola es lisa y plana, de tamaño medio, el lumen es circular y grande. No se observan detalles del yúgulo o del claustro al interior del lumen.

Distribución. Hay un reporte de P. rugosum para el Misisípico de San Salvador Patlanoaya, Puebla, México (Esquivel-Macías et al., 2000). No obstante, su distribución más frecuente es en capas del Pensilvánico-Pérmico, como es el caso del Pensilvánico Inferior (Atokano) de cerros El Tule, Sonora (Buitrón-Sánchez et al., 2012), del Pensilvánico Medio de Sierra Agua Verde, Sonora (Buitrón-Sánchez, Gómez-Espinosa et al., 2007; Buitrón-Sánchez et al., 2015), del Pensilvánico Superior (Virgiliano) de las formaciones Thrifty y Graham de Texas, EE. UU. (Moore y Jeffords, 1968), del Pensilvánico Superior (Misuriano-Virgiliano) de San Salvador Patlanoaya, Puebla (Esquivel-Macías et al., 2000), del Pérmico inferior (Wolfcampiano-Leonardiano) de la Formación Tuzancoa (Arellano-Gil et al., 1998), del Pérmico medio de la Formación Olinalá/Los Arcos, en Olinalá, Guerrero (González-Arreola et al., 1994) y del Pérmico medio de Cerros Los Monos en 
Caborca, Sonora (Buitrón-Sánchez, Almazán-Vázquez et al., 2007).

Número de catálogo: IGM-4875.

\section{Comentarios taxonómicos}

El ejemplar de Las Mesteñas se comparó con P. laeve del Desmoinesiano (Pensilvánico Medio) de Texas (Moore y Jeffords, 1968), pero este último presenta una aréola muy granulosa con cúlmenes más anchos y más cortos que los del ejemplar en estudio. P. rugosum de sierra Las Mesteñas se diferencia de Heterostelechus jeffordsi del Virgiliano (Pensilvánico Superior) de Texas (Moore y Jeffords, 1968) por tener la aréola más delgada y el crenulario más grande. Por otra parte, H. keithi del Pensilvánico Superior de Texas (Moore y Jeffords, 1968) es muy parecido al espécimen de Las Mesteñas, pero el primero tiene la aréola granulosa con el lumen quinquelobulado.

Género Cycloscapus Moore y Jeffords, 1968
Diagnosis: faceta articular con crénulas uniformes, gruesas y rectas formando un crenulario moderadamente ancho, aréola cóncava en forma de tazón, lumen circular relativamente grande.

Especie tipo: Cycloscapus laevis Moore y Jeffords, 1968, pág. 83, lám. 27.

Cycloscapus laevis Moore y Jeffords, 1968

(fig. 11)

Cycloscapus laevis Moore y Jeffords, 1968, pág. 83, lám. 27; Buitrón-Sánchez, Gómez-Espinosa et al., 2007, pág. 204, figura 3 f; Buitrón-Sánchez et al., 2012, pág. 46, figura 7.22

Descripción. La faceta articular tiene forma circular con una epifaceta relativamente conspicua; el crenulario es ancho con crénulas delgadas, la mayoría rectas pero algunas presentan bifurcaciones en los márgenes del crenulario, y los cúlmenes más anchos que las crenelas; la aréola es lisa y estrecha, con la parte interna hundida hacia el interior del lumen, que forma una

Tabla 1

Distribución de las morfoespecies identificadas en la sierra Las Mesteñas, Sonora, México.

\begin{tabular}{|c|c|c|c|}
\hline Especie & Misisípico & Pensilvánico & Pérmico \\
\hline $\begin{array}{l}\text { Pentagonomischus plebeius } \\
\text { Moore y Jeffords, } 1968\end{array}$ & $\begin{array}{l}\text { Formación Paint Creek, } \\
\text { Illinois, EE. UU., Chesteriano } \\
\text { San Salvador Patlanoaya, } \\
\text { Puebla, México, Misisípico } \\
\text { Inferior }\end{array}$ & $\begin{array}{l}\text { Formación Ixtaltepec, Santiago } \\
\text { Ixtaltepec, Oaxaca. } \\
\text { Morrowano-Desmoinesiano }\end{array}$ & \\
\hline $\begin{array}{l}\text { Axilinucrinus angustus } \\
\text { Villanueva-Olea et al., } \\
2011\end{array}$ & & $\begin{array}{l}\text { Formación Ixtaltepec, Santiago } \\
\text { Ixtaltepec, Oaxaca. } \\
\text { Morrowano-Desmoinesiano }\end{array}$ & \\
\hline $\begin{array}{l}\text { Cyclocaudex jucundus Moore } \\
\text { y Jeffords, } 1968\end{array}$ & & $\begin{array}{l}\text { Formación Thrifty de Texas, EE UU. } \\
\text { Virgiliano } \\
\text { San Salvador Patlanoaya, Puebla, } \\
\text { México, Virgiliano } \\
\text { Cerros El Tule, Sonora, México, } \\
\text { Desmoinesiano-Misuriano } \\
\text { Formación Del Monte, Calnali, Hidalgo, } \\
\text { México, Pensilvánico }\end{array}$ & $\begin{array}{l}\text { Formación Guacamaya } \\
\text { de Pemuxco, Hidalgo, } \\
\text { México, Pérmico Inferior } \\
\text { Cerros Los Monos, } \\
\text { Sonora, México, Pérmico } \\
\text { Medio }\end{array}$ \\
\hline $\begin{array}{r}\text { Floricyclus angustimargo } \\
\text { Moore y Jeffords, } 1968\end{array}$ & & $\begin{array}{l}\text { Formación Minturn de Colorado, } \\
\text { EE. UU., Desmoinesiano } \\
\text { San Salvador Patlanoaya, Puebla, } \\
\text { Pensilvánico Medio-Superior }\end{array}$ & \\
\hline $\begin{array}{l}\text { Floricyclus granulosus } \\
\quad \text { Moore y Jeffords, } 1968\end{array}$ & & $\begin{array}{l}\text { Formación Thrifty de Texas, EE. UU., } \\
\text { Virgiliano }\end{array}$ & \\
\hline $\begin{array}{l}\text { Cyclocrista cheneyi Moore } \\
\text { y Jeffords, } 1968\end{array}$ & & $\begin{array}{l}\text { Formación Patlanoaya, San Salvador } \\
\text { Patlanoaya, Puebla, Misuriano-Virgiliano }\end{array}$ & $\begin{array}{l}\text { Texas. Wolfcampiano } \\
\text { Pemuxco, Hidalgo, } \\
\text { Leonardiano }\end{array}$ \\
\hline $\begin{array}{l}\text { Preptopremnum rugosum } \\
\text { Moore y Jeffords, } 1968\end{array}$ & $\begin{array}{l}\text { San Salvador Patlanoaya, } \\
\text { Puebla, Misisípico }\end{array}$ & $\begin{array}{l}\text { Cerros El Tule, Sonora, México, Atokano } \\
\text { Sierra Agua Verde, Sonora, México, } \\
\text { Pensilvánico Medio } \\
\text { Formación Thrifty y Formación Graham } \\
\text { de Texas, EE. UU., Virgiliano } \\
\text { San Salvador Patlanoaya, Puebla, } \\
\text { México, Misuriano-Virgiliano }\end{array}$ & $\begin{array}{l}\text { Formación Tuzancoa, } \\
\text { México, Wolfcampiano- } \\
\text { Leonardiano } \\
\text { Formación Olinalá/Los } \\
\text { Arcos, Guerrero, México, } \\
\text { Pérmico Medio } \\
\text { Cerros Los Monos, } \\
\text { Caborca, Sonora, México, } \\
\text { Pérmico Medio }\end{array}$ \\
\hline $\begin{array}{l}\text { Cycloscapus laevis Moore } \\
\text { y Jeffords, } 1968\end{array}$ & & $\begin{array}{l}\text { Lutita Mingus, Texas, EE. UU., } \\
\text { Desmoinesiano } \\
\text { Sierra Agua Verde, Sonora, México, } \\
\text { Pensilvánico } \\
\text { Cerros El Tule, Sonora, México, Atokano }\end{array}$ & \\
\hline $\begin{array}{c}\text { Cyclocaudiculus regularis } \\
\text { Moore y Jeffords, } 1968\end{array}$ & & $\begin{array}{l}\text { Formación Thrifty, Texas, EE. UU., } \\
\text { Virgiliano }\end{array}$ & \\
\hline
\end{tabular}


concavidad; el lumen es de tamaño medio y aproximadamente circular.

Distribución. C. laevis se ha reportado para el Desmoinesiano (Pensilvánico Medio) de la Lutita Mingus en Texas (Moore y Jeffords, 1968). En México, se distribuye en el Pensilvánico de sierra Agua Verde, Sonora (Buitrón-Sánchez, Gómez-Espinosa et al., 2007) y en el Atokano (Pensilvánico Inferior) de los cerros El Tule, Sonora (Buitrón-Sánchez et al., 2012).

Número de catálogo: IGM-4876.

Comentarios taxonómicos

El material se comparó con Cyclocaudex insaturatus del Pensilvánico Medio de Kansas, EE. UU. (Moore y Jeffords, 1968), pero esta especie tiene una aréola bastante reducida y no presenta un hundimiento hacia la luz del lumen como el ejemplar de Mesteñas. Heterostelechus keithi, del Pensilvánico Superior de Texas (Moore y Jeffords, 1968) comparte el carácter del hundimiento de la aréola, pero presenta una elevación que no es vista en el material de sierra Las Mesteñas.

Género Cyclocaudiculus Moore y Jeffords, 1968

Diagnosis: columna heteromórfica de nodales con una sola cicatriz cirral, faceta articular con crénulas rectas, aréola plana y el lumen circular moderadamente grande.

Especie tipo: Cyclocaudiculus regularis Moore y Jeffords, 1968, pág. 83, lám. 27.

Cyclocaudiculus regularis Moore y Jeffords, 1968

(fig. 12)

Cyclocaudiculus regularis Moore y Jeffords, 1968, pág. 83, lám. 27

Descripción. La placa presenta contorno circular, el crenulario es mediano con 29 crénulas gruesas y rectas, la aréola es plana, con algunas granulaciones debidas probablemente a la conservación del espécimen, el lumen es de tamaño pequeño.

Distribución. C. regularis se reportó para el Virgiliano (Pensilvánico Superior), Formación Thrifty de Texas, EE. UU. (Moore y Jeffords, 1968). El género y la especie se citan por primera vez para México.

Número de catálogo: IGM-4877.

Comentarios taxonómicos

El espécimen de sierra Las Mesteñas se comparó con las diferentes especies del género Heterostelechus (Moore y Jeffords, 1968), pero se descartó su asociación porque el primero no presenta la aréola con una parte elevada, que caracteriza a este último género.

\section{Discusión}

Debido a la escasez de afloramientos del Paleozoico sedimentario en México, la sierra Las Mesteñas representa una región importante, ya que su estudio contribuye al conocimiento integral que hasta ahora se tiene del Paleozoico en el país. Actualmente, se han comenzado prospecciones a detalle, debido a la riqueza y la diversidad de la biota fósil conformada por briozoarios, corales, braquiópodos y crinoides. Estos taxones han sido mencionados en el trabajo de Peiffer-Rangin (1987); no obstante, no se ha publicado ningún estudio taxonómico formal sobre los taxones fósiles que se han recolectado en la sierra Las Mesteñas. Recientemente, también se ha iniciado el estudio de microfacies en esta misma área y los resultados preliminares indican que el depósito ocurrió en un ambiente carbonatado de talud somero, por debajo de la zona arrecifal; a diferencia de lo que ocurre en otras áreas cercanas en el centro de Sonora, como en la sierra Agua Verde (Buitrón-Sánchez, Gómez-Espinosa et al., 2007), donde se ha observado una alternancia de ambientes lagunares de plataforma y de talud.

En total se describieron 8 morfogéneros y 9 morfoespecies, basándose en el trabajo parataxonómico de Moore y Jeffords (1968). Las morfoespecies recolectadas en Las Mesteñas (tabla 1) indican una gran afinidad con las de otras localidades del norte de México (Sonora), del centro (Hidalgo) y del sur (Guerrero, Chiapas), así como de los EE. UU., correspondientes al área del continente medio (Colorado, Texas e Illinois).

La sierra Las Mesteñas se ubica en la región de Cananea que ya ha sido previamente estudiada por diversos autores desde un punto de vista geológico (Bridges, 1965; Gómez-Tagle, 1967; Imlay, 1939; Peiffer-Rangin, 1987; Rangin, 1977; Taliaferro, 1933; Téllez-Girón, 1975; Viveros-Martínez, 1965). Ellos concluyen que las sierras que se ubican en esta área son una extensión de los depósitos de plataforma del sur de Arizona. No obstante, su correlación litológica, particularmente la fauna de crinoides encontrada, no presentó ninguna coincidencia con las especies de crinoides descritas de Arizona, EE. UU.

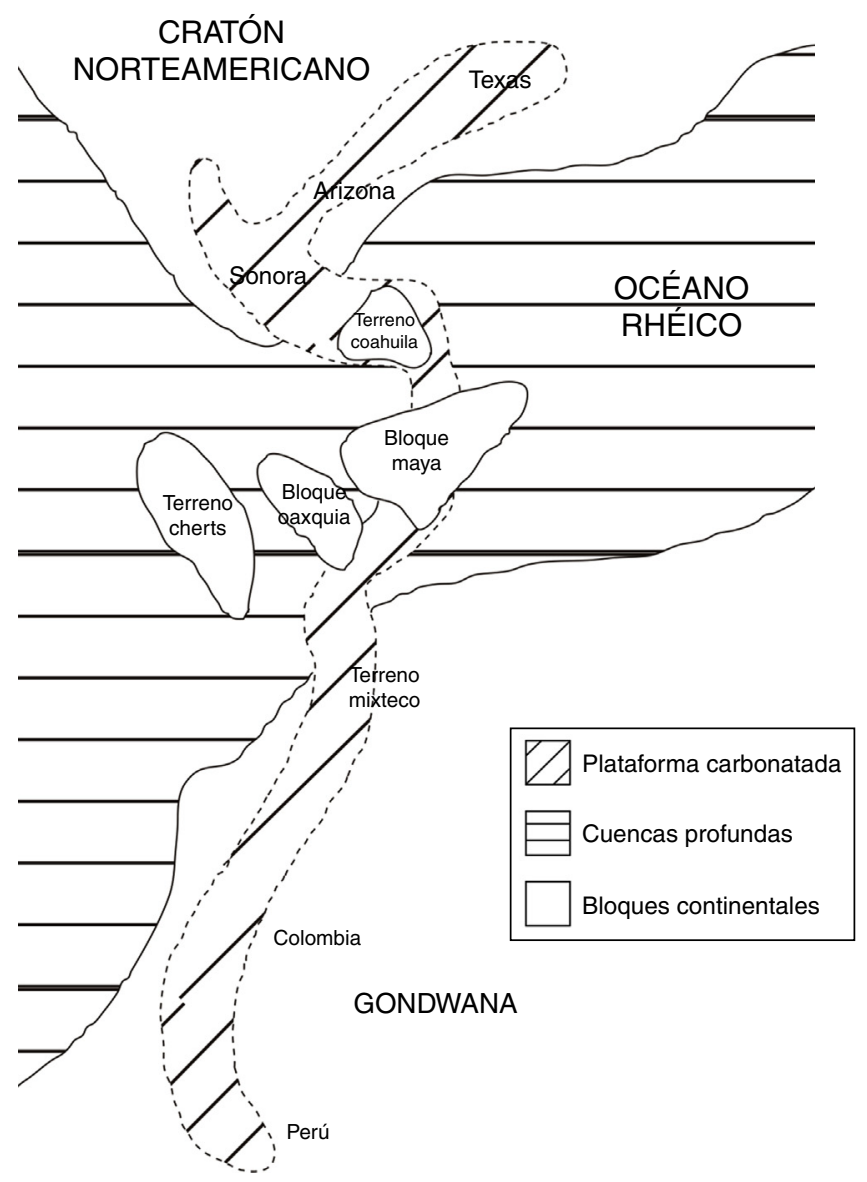

Figura 13. Paleogeografía del Atokano de Sonora a Perú, donde se muestra una plataforma carbonatada hipotética como posible ruta de migración de fusulínidos, chaetétidos y crinoides. Modificado de Almazán-Vázquez et al. (2007). 
En la figura 13 se observa la posición geográfica que posiblemente ocupaba Sonora durante el Paleozoico Superior, al sur de Laurentia. Particularmente, para el Pensilvánico Medio se reporta la existencia de una plataforma carbonatada continua entre Sonora y Sudamérica, vía los bloques de Oaxaquia y Maya. Tal interpretación se basa en la similitud entre la fauna de foraminíferos, principalmente fusulínidos de Sonora y Sudamérica (Almazán-Vázquez et al., 2007; Buitrón-Sánchez, Gómez-Espinosa et al., 2007; Buitrón-Sánchez et al., 2012). Este corredor de aguas poco profundas habría servido como puente de migración para las faunas de fusulínidos encontradas en el norte (Sonora, Arizona y Texas) con las del sur (Sudamérica).

En algunos casos, los crinoides debieron seguir un patrón de dispersión similar desde el norte hasta el sur a través de este corredor de plataformas carbonatadas. Igualmente, debió existir una conexión entre Sonora y el «midcontinent» del Pensilvánico, por la correlación de faunas encontradas en ambas áreas. Esta idea es también apoyada por el hecho de hallar las mismas morfoespecies del Misisípico de EE. UU. («midcontinent») en el Pensilvánico del sur de México, como P. plebeius (tabla 1); o bien, del Pensilvánico de algunas áreas de EE. UU. y en el Pensilvánico-Pérmico de México, como C. jucundus (tabla 1).

\section{Agradecimientos}

RVO agradece al posgrado en Ciencias Biológicas de la Universidad Nacional Autónoma de México y al Conacyt por la beca otorgada. La investigación se llevó a cabo en el marco de los Proyectos Conacyt 165826, 235970 y ECOS Francia y Conacyt México Núm. M13U01 «Evolución de los Ecosistemas del Paleozoico de México y su biodiversidad».

\section{Referencias}

Almazán-Vázquez, E., Buitrón-Sánchez, B. E., Vachard, D., Mendoza-Madera, C. y Gómez-Espinosa, C. (2007). The late Atokan (Moscovian, Pennsylvanian) chaetetid accumulations of Sierra Agua Verde, Sonora (NW Mexico): composition, facies and palaeoenvironmental signals. En J. J. Álvaro, M. Aretz, F. Boulvain, A. Munnecke, D. Vachard, y E. Vennin (Eds.), Palaeozoic reefs and bioaccumulations: climatic and evolutionary controls (275) (pp. 189-200). London: Geological Society, Special Publications.

Arellano-Gil, J., Vachard, D., Yussim, S. y Flores de Dios-González, A. (1998). Aspectos estratigráficos, estructurales y paleogeográficos del Pérmico Inferior al Jurásico Inferior en Pemuxco, estado de Hidalgo, México. Revista Mexicana de Ciencias Geológicas, 15, 9-13.

Bridges, L. W. (1965). Geología del área de Plomosas, Chihuahua. Boletín del Instituto de Geología. UNAM, 84, 1-34.

Buitrón-Sánchez, B. E. y Solís-Marín, F. A. (1993). La biodiversidad en los equinodermos fósiles y recientes de México. Revista de la Sociedad Mexicana de Historia Natural, Esp., 44, 209-231.

Buitrón-Sánchez, B. E., Patiño, J. y Moreno, A. (1987). Crinoides del Paleozoico Tardío de Calnali, Hidalgo. Revista de la Sociedad Mexicana de Paleontología, 1, 125-136.

Buitrón-Sánchez, B. E., Gómez-Espinosa, C., Almazán-Vázquez, E. y Vachard, D. (2007). A late Atokan regional encrinite (early late Moscovian, Middle Pennsylvanian) in the Sierra Agua Verde, Sonora State, NW Mexico. En J. J. Álvaro, M. Aretz, F. Boulvain, A. Munnecke, D. Vachard, y E. Vennin (Eds.), Palaeozoic reefs and bioaccumulations: climatic and evolutionary controls (275) (pp. 201-209). London: Geological Society, Special Publications.

Buitrón-Sánchez, B. E., Almazán-Vázquez, E. y Vachard, D. (2007). Middle Permian crinoids (Echinodermata, Crinoidea) from cerros Los Monos, Caborca,
Sonora. Mexico and paleogeographic considerations. Revista Mexicana de Ciencias Geológicas, 24, 344-353.

Buitrón-Sánchez, B. E., Gómez-Espinosa, C., Almazán-Vázquez, E., Vachard, D., Laguarda-Figueras, A. y Solís-Marín, F. (2008). A review of the crinoid columnals (Echinodermata-Crinoidea) from the Carboniferous of Mexico. Revista de Biología Tropical, 56, 1-12.

Buitrón-Sánchez, B. E., Vachard, D., Almazán-Vázquez, E. y Palafox-Reyes, J. J. (2012). Una secuencia cratónica del Carbonífero al Pérmico inferior expuesta en los Cerros El Tule, noreste de Sonora, México. Revista Mexicana de Ciencias Geológicas, 29, 39-62.

Buitrón-Sánchez, B. E., Chacón-Wences, O., Vachard, D., Palafox-Reyes, J. J., Jiménez-López, J. C. y Sour-Tovar, F. (2015). Pennsylvanian biota of The Sierra Agua Verde, Sonora, Mexico: biostratigraphic and paleogeographic considerations. Revista Mexicana de Biodiversidad, 86, 521-527.

Esquivel-Macías, C., Ausich, W. I., Buitrón-Sánchez, B. E. y Flores de Dios, A. (2000). Pennsylvanian and Mississippian pluricolumnal assemblages (Class Crinoidea) from southern Mexico and a new occurrence of a column with a tetralobate lumen. Journal of Paleontology, 74, 1187-1190.

Esquivel-Macías, C., Solís-Marín, F. y Buitrón-Sánchez, B. E. (2004). Nuevos registros de placas columnares de crinoideos (Echinodermata, Crinoidea) del Paleozoico Superior de México, algunas implicaciones paleobiogeográficas y paleoambientales. Coloquios de Paleontología, 54, 15-23.

Gómez-Tagle, A. (1967). Estudio geológico de la sierra Las Mesteñas, Municipio de Fronteras, Sonora. Tesis. Facultad de Ingeniería, Universidad Nacional Autónoma de México. México, D.F.

González-Arreola, C., Villaseñor-Martínez, A. B. y Corona-Esquivel, R. (1994). Permian fauna of the Los Arcos Formation, municipality of Olinalá, State of Guerrero, Mexico. Revista Mexicana de Ciencias Geológicas, 11, 214-221.

Imlay, W. R. (1939). Paleogeographic studies in northeastern Sonora. Geological Society of America Bulletin, 50, 1723-1744.

Moore, R. C. y Jeffords, R. M. (1968). Classification and nomenclature of fossil crinoids based on studies of dissociated parts of their columns. University of Kansas Paleontological Contributions, Echinodermata, 9, 1-86.

Moreno-Cano, A. y Patiño-Ruiz, J. (1981). Estudio paleozoico en la región de Calnali, Hidalgo (en la sierra Madre Oriental). Tesis. Escuela Superior de Ingeniería y Arquitectura, Instituto Politécnico Nacional. México, D.F.

Page, W. R., Gray, F., Iriondo, A., Miggins, D., Blodgett, R. B., Maldonado, F., et al. (2010). Stratigraphy and Mesozoic-Cenozoic tectonic history of northern sierra Los Ajos and adjacent areas, Sonora, Mexico. Journal of South American Earth Sciences, 29, 557-571.

Peiffer-Rangin, F. (1987). Biostratigraphic study of Paleozoic rocks of northeastern and central Sonora. París, Francia: Université de Paris [Tesis].

Rangin, C. (1977). Tectónicas sobrepuestas en Sonora septentrional. Revista del Instituto de Geología, UNAM, 1, 44-47.

Ransome, F.L. (1904). The geology and ore deposits of the Bisbee Quadrangle, Arizona. Washington: U.S. Geological Survey, Professional Paper, 21.

Stewart J. H., McMenamin, M.A. S. y Morales-Ramírez, J.M. (1984). Upper Proterozoic and Cambrian rocks in the Caborca region, Sonora, MéxicoPhysical stratigraphy, biostratigraphy, paleocurrent studies, and regional relations. Washington: U.S. Geological Survey, Professional Paper, 1309.

Taliaferro, N. L. (1933). An occurrence of Upper Cretaceous sediments in northern Sonora Mexico. The Journal of Geology, 41, 12-37.

Téllez-Girón, C. (1975). Microfacies y microfósiles del Pérmico de las sierras de Los Chinos, Sta. Rita y La Salada, Chihuahua y la sierra de Teras, Sonora. México, D.F: Instituto Mexicano del Petróleo, Subdirección de Tecnología de Exploración.

Ubaghs, G., Moore, R. C., Wienberg-Rasmussen, H., Lane, N. G., Breimer, A., Strimple, H. L., et al. (1978). Part T Echinodermata 2. En R. C. Moore y C. Teichert (Eds.), Treatise on Invertebrate Paleontology (Vol. 1) (pp. T1-T401). Kansas: University of Kansas and the Geological Society of America.

Velasco-de León, M. P. y Buitrón-Sánchez, B. E. (1992). Algunos crinoides (Echinodermata-Crinoidea) del Misisípico-Pensilvánico de San Salvador Patlanoaya, estado de Puebla. Revista de la Sociedad Mexicana de Paleontología, 5, 71-81.

Villanueva-Olea, R., Castillo-Espinoza, K. M., Sour-Tovar, F., Quiroz-Barroso, S. A. y Buitrón-Sánchez, B. E. (2011). Placas columnares de crinoides del 
Carbonífero de la región de Santiago Ixtaltepec, Municipio de Nochixtlán. Oaxaca; consideraciones estratigráficas y paleobiogeográficas. Boletín de la Sociedad Geológica Mexicana, 63, 443-457.

Villaseñor-Martínez, A. B., Martínez-Cortés, A., Contreras y Montero, B. (1987). Bioestratigrafía del Paleozoico Superior de San Salvador
Patlanoaya, Puebla, México. Revista de la Sociedad Mexicana de Paleontología, 1, 396-417.

Viveros-Martínez, A. (1965). Estudio geológico de la sierra de Cabullona municipio de Agua Prieta, estado de Sonora. Tesis de maestría. Facultad de Ingeniería, Universidad Nacional Autónoma de México. México, D.F. 